\title{
Efficient Fluoride Selective Fluoresent Host: Experiment and Theory
}

\author{
Jin Yong Lee*, Eun Jin Cho, Shaul Mukamel ${ }^{\dagger *}$, and Kye Chun Nam* \\ Department of Chemistry, Chonnam National University, 300 Yongbong-Dong, Bukgu, \\ Kwangju 500-757, Korea, Department of Chemistry, University of California, Irvine, \\ Irvine, CA 92697, USA \\ E-mail: jinyong@chonnam.ac.kr
}

Supporting Information

\section{Contents}

Total energies of F-, Cl-, Br-, 1, 1F-, 1Cl-, and 1Br-. (Table S1, in plain text)

Number of imaginary frequencies of $\mathbf{1}$ and $\mathbf{1 F}$-. (Table S2, in plain text)

Cartesian coordinates of $\mathbf{1}$. (Table S3, in plain text).

Cartesian coordinates of 1F-. (Table S4, in plain text).

Cartesian coordinates of 1Cl-. (Table S5, in plain text).

Cartesian coordinates of $\mathbf{1 B r}-$. (Table S6, in plain text). 
Table S1

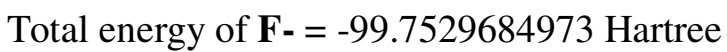

Total energy of Cl- $=-460.248731620$ Hartree

Total energy of Br- $=-2571.46436594$ Hartree

Total energy of $\mathbf{1}=-1296.08269153$

Total energy of 1F- $=-1396.0407401$

Total energy of $\mathbf{1 C l}-=-1756.42079674$

Total energy of $\mathbf{1 B r}-=-3867.63571251$

Table S2

\# of imaginary frequencies of $\mathbf{1}=0$

\# of imaginary frequencies of $\mathbf{1 F}-=0$ 
Table S3. Cartesian coordinates of $\mathbf{1}$

\begin{tabular}{|c|c|c|c|c|c|}
\hline Number & Atomic number & & $\mathrm{x}$ & $\mathrm{y}$ & $\mathrm{z}$ \\
\hline 1 & 6 & 0 & -3.258648 & 0.097141 & 0.037340 \\
\hline 2 & 6 & 0 & -4.691832 & 0.185739 & -0.068378 \\
\hline 3 & 6 & 0 & -2.704830 & -1.192218 & 0.362156 \\
\hline 4 & 6 & 0 & -2.505254 & 1.305361 & -0.192061 \\
\hline 5 & 6 & 0 & -3.508442 & -2.315719 & 0.416424 \\
\hline 6 & 6 & 0 & -3.147641 & 2.518594 & -0.355267 \\
\hline 7 & 6 & 0 & -4.898043 & -2.220224 & 0.203188 \\
\hline 8 & 6 & 0 & -4.556044 & 2.594371 & -0.347935 \\
\hline 9 & 6 & 0 & -5.481366 & -0.992050 & 0.002371 \\
\hline 10 & 6 & 0 & -5.310858 & 1.451635 & -0.244212 \\
\hline 11 & 1 & 0 & -3.054713 & -3.270333 & 0.652193 \\
\hline 12 & 1 & 0 & -2.553301 & 3.409727 & -0.505393 \\
\hline 13 & 1 & 0 & -5.505665 & -3.119536 & 0.245904 \\
\hline 14 & 1 & 0 & -5.034096 & 3.561841 & -0.472047 \\
\hline 15 & 1 & 0 & -6.558765 & -0.899809 & -0.106236 \\
\hline 16 & 1 & 0 & -6.395692 & 1.495077 & -0.293606 \\
\hline 17 & 7 & 0 & -1.333616 & -1.304124 & 0.717930 \\
\hline 18 & 7 & 0 & -1.088449 & 1.213293 & -0.327863 \\
\hline 19 & 1 & 0 & -0.934107 & -0.450108 & 1.080527 \\
\hline 20 & 1 & 0 & -0.775176 & 0.450247 & -0.912645 \\
\hline 21 & 6 & 0 & -0.444878 & -2.235492 & 0.190682 \\
\hline 22 & 6 & 0 & -0.150495 & 2.204963 & -0.035846 \\
\hline 23 & 8 & 0 & -0.774106 & -3.170780 & -0.520725 \\
\hline 24 & 8 & 0 & -0.430822 & 3.289699 & 0.450706 \\
\hline 25 & 7 & 0 & 0.871112 & -1.938333 & 0.553960 \\
\hline 26 & 7 & 0 & 1.138640 & 1.780846 & -0.329861 \\
\hline 27 & 1 & 0 & 0.986493 & -1.289202 & 1.322221 \\
\hline 28 & 1 & 0 & 1.256183 & 0.821006 & -0.628744 \\
\hline 29 & 6 & 0 & 2.039794 & -2.676630 & 0.249580 \\
\hline 30 & 6 & 0 & 2.348481 & 2.494894 & -0.181305 \\
\hline 31 & 6 & 0 & 3.225257 & -2.284837 & 0.893850 \\
\hline 32 & 6 & 0 & 4.425039 & -2.939122 & 0.624829 \\
\hline 33 & 6 & 0 & 4.462794 & -3.992397 & -0.290752 \\
\hline 34 & 6 & 0 & 3.283540 & -4.377841 & -0.929780 \\
\hline
\end{tabular}




$\begin{array}{rrrrrr}35 & 6 & 0 & 2.072670 & -3.732805 & -0.673477 \\ 36 & 6 & 0 & 3.537035 & 1.787480 & -0.427471 \\ 37 & 6 & 0 & 4.771396 & 2.422262 & -0.316896 \\ 38 & 6 & 0 & 4.841819 & 3.769661 & 0.042134 \\ 39 & 6 & 0 & 3.659013 & 4.468041 & 0.288918 \\ 40 & 6 & 0 & 2.412988 & 3.848852 & 0.181750 \\ 41 & 1 & 0 & 3.206207 & -1.463691 & 1.608557 \\ 42 & 1 & 0 & 5.329996 & -2.622252 & 1.136087 \\ 43 & 1 & 0 & 5.396967 & -4.504635 & -0.501448 \\ 44 & 1 & 0 & 3.296912 & -5.197268 & -1.643611 \\ 45 & 1 & 0 & 1.160901 & -4.040783 & -1.165025 \\ 46 & 1 & 0 & 3.493447 & 0.734986 & -0.702930 \\ 47 & 1 & 0 & 5.678940 & 1.857641 & -0.513014 \\ 48 & 1 & 0 & 5.803779 & 4.266319 & 0.129150 \\ 49 & 1 & 0 & 3.697632 & 5.516966 & 0.571048 \\ 50 & 1 & 0 & 1.500701 & 4.392741 & 0.380938\end{array}$


Table S4. Cartesian coordinates of 1F-

\begin{tabular}{|c|c|c|c|c|}
\hline Number & Atomic number & $\mathrm{x}$ & $\mathrm{y}$ & $\mathrm{Z}$ \\
\hline 1 & 6 & 3.083492 & -0.292337 & 0.089682 \\
\hline 2 & 6 & 4.524808 & -0.403608 & 0.075495 \\
\hline 3 & 6 & 2.332901 & -1.452487 & -0.366821 \\
\hline 4 & 6 & 2.534393 & 0.970648 & 0.535208 \\
\hline 5 & 6 & 3.007886 & -2.589230 & -0.804772 \\
\hline 6 & 6 & 3.377501 & 2.006650 & 0.910171 \\
\hline 7 & 6 & 4.410887 & -2.663592 & -0.784978 \\
\hline 8 & 6 & 4.776402 & 1.877763 & 0.874612 \\
\hline 9 & 6 & 5.160627 & -1.599404 & -0.350945 \\
\hline 10 & 6 & 5.336950 & 0.691124 & 0.472956 \\
\hline 11 & 1 & 2.416870 & -3.423614 & -1.153713 \\
\hline 12 & 1 & 2.926121 & 2.940314 & 1.220828 \\
\hline 13 & 1 & 4.893716 & -3.577371 & -1.124869 \\
\hline 14 & 1 & 5.401773 & 2.715703 & 1.173831 \\
\hline 15 & 1 & 6.247298 & -1.642072 & -0.337291 \\
\hline 16 & 1 & 6.417170 & 0.565906 & 0.440965 \\
\hline 17 & 7 & 0.934444 & -1.434844 & -0.355718 \\
\hline 18 & 7 & 1.138647 & 1.216384 & 0.588916 \\
\hline 19 & 1 & 0.503861 & -0.567222 & -0.069263 \\
\hline 20 & 6 & 0.048478 & -2.516844 & -0.442602 \\
\hline 21 & 6 & 0.545378 & 2.362975 & 0.051399 \\
\hline 22 & 8 & 0.328220 & -3.616767 & -0.920827 \\
\hline 23 & 8 & 1.161219 & 3.254023 & -0.530330 \\
\hline 24 & 7 & -1.173816 & -2.156708 & 0.077067 \\
\hline 25 & 7 & -0.823030 & 2.332771 & 0.253921 \\
\hline 26 & 1 & -1.186410 & -1.272525 & 0.650366 \\
\hline 27 & 1 & -1.162768 & 1.467865 & 0.729975 \\
\hline 28 & 6 & -2.343899 & -2.912647 & 0.111894 \\
\hline 29 & 6 & -1.758465 & 3.298840 & -0.104075 \\
\hline 30 & 6 & -3.417858 & -2.354800 & 0.837814 \\
\hline 31 & 6 & -4.636735 & -3.018427 & 0.919514 \\
\hline 32 & 6 & -4.820436 & -4.250852 & 0.283280 \\
\hline 33 & 6 & -3.756671 & -4.803973 & -0.432558 \\
\hline 34 & 6 & -2.524706 & -4.154666 & -0.525332 \\
\hline
\end{tabular}




$\begin{array}{rrrrr}35 & 6 & -3.104992 & 3.035991 & 0.225878 \\ 36 & 6 & -4.107977 & 3.946127 & -0.091389 \\ 37 & 6 & -3.801853 & 5.143927 & -0.744166 \\ 38 & 6 & -2.469995 & 5.406545 & -1.072353 \\ 39 & 6 & -1.450691 & 4.505560 & -0.764156 \\ 40 & 1 & -3.257255 & -1.399189 & 1.331006 \\ 41 & 1 & -5.450202 & -2.568827 & 1.485369 \\ 42 & 1 & -5.774004 & -4.769554 & 0.346841 \\ 43 & 1 & -3.881551 & -5.763481 & -0.931176 \\ 44 & 1 & -1.698905 & -4.590807 & -1.070635 \\ 45 & 1 & -3.346221 & 2.104917 & 0.733091 \\ 46 & 1 & -5.137667 & 3.715254 & 0.173987 \\ 47 & 1 & -4.585593 & 5.855238 & -0.993104 \\ 48 & 1 & -2.211670 & 6.332541 & -1.582912 \\ 49 & 1 & -0.421162 & 4.708757 & -1.024458 \\ 50 & 9 & -1.034353 & 0.035554 & 1.451522 \\ 51 & 1 & 0.494007 & 0.647219 & 1.174888\end{array}$


Table S5. Cartesian coordinates of $\mathbf{1 C l}-$

\begin{tabular}{|c|c|c|c|c|c|}
\hline Number & Atomic number & & $\mathrm{x}$ & $\mathrm{y}$ & $\mathrm{z}$ \\
\hline 1 & 6 & 0 & 3.103675 & -0.536348 & 0.054589 \\
\hline 2 & 6 & 0 & 4.532789 & -0.738011 & -0.010274 \\
\hline 3 & 6 & 0 & 2.259284 & -1.668401 & -0.291024 \\
\hline 4 & 6 & 0 & 2.654667 & 0.777079 & 0.442737 \\
\hline 5 & 6 & 0 & 2.842512 & -2.869475 & -0.685315 \\
\hline 6 & 6 & 0 & 3.564878 & 1.777769 & 0.736448 \\
\hline 7 & 6 & 0 & 4.238590 & -3.027631 & -0.732007 \\
\hline 8 & 6 & 0 & 4.952462 & 1.561324 & 0.665069 \\
\hline 9 & 6 & 0 & 5.074018 & -1.991728 & -0.397314 \\
\hline 10 & 6 & 0 & 5.422176 & 0.323794 & 0.301701 \\
\hline 11 & 1 & 0 & 2.190190 & -3.685444 & -0.958904 \\
\hline 12 & 1 & 0 & 3.181035 & 2.753507 & 1.010733 \\
\hline 13 & 1 & 0 & 4.646731 & -3.988103 & -1.038809 \\
\hline 14 & 1 & 0 & 5.638640 & 2.371168 & 0.900055 \\
\hline 15 & 1 & 0 & 6.155100 & -2.104019 & -0.430195 \\
\hline 16 & 1 & 0 & 6.490925 & 0.130772 & 0.238568 \\
\hline 17 & 7 & 0 & 0.868788 & -1.549201 & -0.193529 \\
\hline 18 & 7 & 0 & 1.264123 & 1.105806 & 0.531290 \\
\hline 19 & 1 & 0 & 0.535499 & -0.631613 & 0.078167 \\
\hline 20 & 6 & 0 & -0.102921 & -2.523259 & -0.417687 \\
\hline 21 & 6 & 0 & 0.745152 & 2.229261 & -0.131701 \\
\hline 22 & 8 & 0 & 0.093559 & -3.579887 & -1.016278 \\
\hline 23 & 8 & 0 & 1.376298 & 2.865280 & -0.969288 \\
\hline 24 & 7 & 0 & -1.320608 & -2.137552 & 0.111667 \\
\hline 25 & 7 & 0 & -0.553080 & 2.490560 & 0.256010 \\
\hline 26 & 1 & 0 & -1.304777 & -1.400101 & 0.830642 \\
\hline 27 & 1 & 0 & -0.953949 & 1.854221 & 0.958393 \\
\hline 28 & 6 & 0 & -2.549749 & -2.799288 & -0.026066 \\
\hline 29 & 6 & 0 & -1.395262 & 3.510917 & -0.198665 \\
\hline 30 & 6 & 0 & -3.621177 & -2.315589 & 0.751146 \\
\hline 31 & 6 & 0 & -4.881386 & -2.897409 & 0.654005 \\
\hline 32 & 6 & 0 & -5.108503 & -3.968865 & -0.214655 \\
\hline 33 & 6 & 0 & -4.046235 & -4.447218 & -0.983221 \\
\hline 34 & 6 & 0 & -2.774287 & -3.879098 & -0.899690 \\
\hline
\end{tabular}




$\begin{array}{rrrrrr}35 & 6 & 0 & -2.685303 & 3.567614 & 0.365722 \\ 36 & 6 & 0 & -3.586124 & 4.552883 & -0.026252 \\ 37 & 6 & 0 & -3.228446 & 5.504258 & -0.985904 \\ 38 & 6 & 0 & -1.950766 & 5.446792 & -1.545345 \\ 39 & 6 & 0 & -1.033077 & 4.466275 & -1.166426 \\ 40 & 1 & 0 & -3.444344 & -1.482645 & 1.426959 \\ 41 & 1 & 0 & -5.692545 & -2.506858 & 1.264636 \\ 42 & 1 & 0 & -6.094085 & -4.422130 & -0.288935 \\ 43 & 1 & 0 & -4.202105 & -5.281939 & -1.663906 \\ 44 & 1 & 0 & -1.953062 & -4.264087 & -1.487255 \\ 45 & 1 & 0 & -2.966018 & 2.827928 & 1.111485 \\ 46 & 1 & 0 & -4.576432 & 4.574574 & 0.423361 \\ 47 & 1 & 0 & -3.933355 & 6.273390 & -1.291828 \\ 48 & 1 & 0 & -1.654594 & 6.177377 & -2.295546 \\ 49 & 1 & 0 & -0.045173 & 4.422004 & -1.602892 \\ 50 & 17 & 0 & -1.229388 & 0.211479 & 2.402019 \\ 51 & 1 & 0 & 0.746689 & 0.802545 & 1.368185\end{array}$


Table S6. Cartesian coordinates of $\mathbf{1 B r}$ -

\begin{tabular}{|c|c|c|c|c|c|}
\hline Number & Atomic number & & $\mathrm{X}$ & $\mathrm{y}$ & $\mathrm{z}$ \\
\hline 1 & 6 & 0 & 3.230471 & -0.392906 & -0.042941 \\
\hline 2 & 6 & 0 & 4.669330 & -0.520006 & -0.059441 \\
\hline 3 & 6 & 0 & 2.457804 & -1.570505 & -0.403098 \\
\hline 4 & 6 & 0 & 2.700978 & 0.898120 & 0.316262 \\
\hline 5 & 6 & 0 & 3.114477 & -2.743788 & -0.763158 \\
\hline 6 & 6 & 0 & 3.546907 & 1.948733 & 0.626360 \\
\hline 7 & 6 & 0 & 4.517823 & -2.829310 & -0.762486 \\
\hline 8 & 6 & 0 & 4.945606 & 1.805264 & 0.601525 \\
\hline 9 & 6 & 0 & 5.287110 & -1.747773 & -0.413845 \\
\hline 10 & 6 & 0 & 5.490999 & 0.590434 & 0.268254 \\
\hline 11 & 1 & 0 & 2.514534 & -3.595510 & -1.047558 \\
\hline 12 & 1 & 0 & 3.103572 & 2.905377 & 0.878054 \\
\hline 13 & 1 & 0 & 4.985033 & -3.770357 & -1.044045 \\
\hline 14 & 1 & 0 & 5.580434 & 2.652560 & 0.848375 \\
\hline 15 & 1 & 0 & 6.373046 & -1.803804 & -0.410939 \\
\hline 16 & 1 & 0 & 6.569873 & 0.453609 & 0.241699 \\
\hline 17 & 7 & 0 & 1.060657 & -1.519237 & -0.354666 \\
\hline 18 & 7 & 0 & 1.291400 & 1.151506 & 0.362922 \\
\hline 19 & 1 & 0 & 0.677247 & -0.614266 & -0.105482 \\
\hline 20 & 6 & 0 & 0.146101 & -2.540462 & -0.604109 \\
\hline 21 & 6 & 0 & 0.734117 & 2.234231 & -0.337583 \\
\hline 22 & 8 & 0 & 0.416172 & -3.595330 & -1.176114 \\
\hline 23 & 8 & 0 & 1.350283 & 2.872585 & -1.184004 \\
\hline 24 & 7 & 0 & -1.110411 & -2.204926 & -0.133396 \\
\hline 25 & 7 & 0 & -0.579269 & 2.450606 & 0.024410 \\
\hline 26 & 1 & 0 & -1.159368 & -1.453366 & 0.567114 \\
\hline 27 & 1 & 0 & -0.963044 & 1.825295 & 0.745430 \\
\hline 28 & 6 & 0 & -2.300818 & -2.927860 & -0.305576 \\
\hline 29 & 6 & 0 & -1.459236 & 3.419967 & -0.470324 \\
\hline 30 & 6 & 0 & -3.419699 & -2.493361 & 0.432300 \\
\hline 31 & 6 & 0 & -4.645917 & -3.136646 & 0.295564 \\
\hline 32 & 6 & 0 & -4.790386 & -4.221982 & -0.573521 \\
\hline 33 & 6 & 0 & -3.680270 & -4.652024 & -1.301913 \\
\hline 34 & 6 & 0 & -2.441239 & -4.021680 & -1.178975 \\
\hline
\end{tabular}




$\begin{array}{lccccc}35 & 6 & 0 & -2.752852 & 3.444505 & 0.087938 \\ 36 & 6 & 0 & -3.690836 & 4.376960 & -0.343934 \\ 37 & 6 & 0 & -3.367127 & 5.305442 & -1.337504 \\ 38 & 6 & 0 & -2.085538 & 5.279519 & -1.890240 \\ 39 & 6 & 0 & -1.130586 & 4.352002 & -1.471741 \\ 40 & 1 & 0 & -3.304435 & -1.652477 & 1.111986 \\ 41 & 1 & 0 & -5.495342 & -2.783845 & 0.876328 \\ 42 & 1 & 0 & -5.749543 & -4.723286 & -0.678539 \\ 43 & 1 & 0 & -3.771706 & -5.496811 & -1.981853 \\ 44 & 1 & 0 & -1.582631 & -4.369067 & -1.735748 \\ 45 & 1 & 0 & -3.005594 & 2.722074 & 0.860324 \\ 46 & 1 & 0 & -4.683341 & 4.375305 & 0.101244 \\ 47 & 1 & 0 & -4.101147 & 6.033244 & -1.674481 \\ 48 & 1 & 0 & -1.815783 & 5.993106 & -2.666275 \\ 49 & 1 & 0 & -0.139310 & 4.332129 & -1.902367 \\ 50 & 35 & 0 & -1.258407 & 0.160369 & 2.376858 \\ 51 & 1 & 0 & 0.780170 & 0.852232 & 1.205854\end{array}$

